\title{
Social Participation as a goal of the post-stroke rehabilitation program: a literature review
}

\author{
Felipe Heylan Nogueira de Souza', Edson Meneses da Silva Filho', Leandro Gonçalves Cezarino', \\ Egmar Longo Araújo de Melo', Ênio Walker Azevedo Cacho'
}

\begin{abstract}
Introduction: Stroke is considered one of the leading causes of long-term disability worldwide. Physical, cognitive and psychological disabilities resulting from stroke can lead to a wide variety of activity limitations and participations restrictions. Objective: To verify the incidence of articles in the literature that used physiotherapy programs aimed at social participation and to analyze if these programs are based on domains of the International Classification of Functioning Disability and Health (ICF) in post-stroke people. Method: It was selected any type of study that presented post-stroke human sample over 18 years of age with no gender restriction, to be written in any language and year of publication, to have used physical rehabilitation as a form of treatment and social participation assessed by ICF as outcome. The descriptors and Boolean operators: stroke, rehabilitation, International Classification of Functioning Disability and Health, social participation, were used in the sample search strategy in database PEDro, PubMed, Web of Science and Scielo. Results: After applying the inclusion/exclusion criteria, five articles remaining. Assessment tools for participation in daily and social activities were not consensual in the range of areas that needed to be addressed in stroke rehabilitation. A single study used the ICF-based design model in its program. Conclusion: There are few studies that have measured the various characteristics of social participation using the ICF as a tool. More studies are needed to create an ideal standardization and strategy that direct interventions to improve specific aspects of social participation, including the involvement in activities that provide post-stroke individuals interaction with society.
\end{abstract}

Keywords: Stroke; International Classification of Functioning, Disability and Health; Social Participation.

\section{INTRODUCTION}

Described as a multidimensional concept, the social participation is defined by the International Classification of Functioning, Disability and Health (ICF) as involvement of a person in a real-life situation, representing a social perspective of functionality ${ }^{(1,2)}$. Social participation is considered as one of the most relevant fundamental results to a successful recovery, however, individuals after cerebrovascular accident (CVA) have shown dissatisfaction with their own ability to develop some of its aspects such as socializing, tours and travelling(3), so the ICF considers that the functionality is the result of complex interactions, among the health and environmental measures as well as personal factors conditions ${ }^{(1)}$.

Corroborating the ICF, some authors state that several factors may affect the social participation, such as, the restrictions due to changes in functionality, limitations in activities and the interference of environmental and personal factors $^{(2,4)}$. Among the physical and social consequences, those which are generated by the stroke are one of the important health problems in industrialized countries ${ }^{(5)}$. Those impacts on functionality may result in neurological and functional changes, in which generate limitations in daily live activities of these subjects ${ }^{(4,6)}$.

The consequences of stroke and the complex recovery process often require the necessity for various therapeutic interventions ${ }^{(7,8)}$. The knowledge regarding the treatments which promote the best results may help optimize its effects and provide a better functionality for these patients ${ }^{(2)}$.

The successful recovery of a stroke should result in a quality of life and degree of participation in the community similar to the previous injury, however, not all patients have access to care and rehabilitation that they need ${ }^{(9,10)}$. The provision of rehabilitation services after discharge is essential to promote independence and reintegration of patients in the community ${ }^{(10)}$. Although many interventions and measurements are not aimed at social participation, even though it is considered one of the most important and fundamental factors for a successful recovery, there is the necessity to measure what the studies have been using to

Corresponding author: Edson Meneses da Silva Filho Rua Vila Trairi, S/N; Centro; Santa Cruz (RN), Brazil. CEP: $59200-000$. Phone:+55 (81) 9 9470 6661; +55 (81) 36311304 Email: meneses.edson@yahoo.com.br

1 Universidade Federal do Rio Grande do Norte (UFRN), Santa Cruz (RN), Brazil.

Financial support: None. 
assess the social participation of people who had stroke, in order to suggest a standardization of the use of more sensitive tools to classify the social participation in this population. Therefore, and present study aimed to determine the incidence of articles which used physical therapy programs for the social participation and analyze if these programs are based on the ICF domains in people who had stroke.

\section{METHODS}

The present review followed the recommendations of Preferred Reporting Items for Systematic Reviews and Meta-Analyzes (PRISMA)(11).

\section{Eligibility criteria}

The articles were selected according to the following inclusion criteria: any study which presented in the sample, human aged 18 years old, who had cerebrovascular accident, no gender restriction, to be written in any language and year of publication, to be used as a treatment the physical rehabilitation and as outcome the social participation assessed by the International Classification of Functioning Disability and Health (ICF). The descriptors (Mesh/Decs) and the Booleans operators were used in accordance with the PICO: P- stroke; I- rehabilitation, International Classification of functioning disability and health; O- Social participation.

A simple search strategy was performed according to the specifics of databases by using the Booleans keywords and operators: stroke AND rehabilitation OR International Classification of functioning, disability and health AND Social participation. The used databases were the PEDro, PubMed, Web of Science and Scielo. To identify further published, unpublished and ongoing studies, a search was performed on clinical trial records, reference lists of included studies, and contact was made with the authors, if necessary. The last search was conducted in July/2017.

\section{Selection of studies}

To decide which studies would be part of the present review, two researchers independently, after the search, read the titles, abstracts and full articles to select those which would meet the inclusion criteria. After making the selection, the items have been stored so that in the consensus meeting it was decided which of them would be part in the study. If there was disagreement between the researchers, a third researcher would disown it.

\section{Data collection process}

Study data were also collected independently by two researchers. A form has been created by researchers in an attempt to obtain the necessary data containing: type of study, sample (country, age and gender), stage of disease, intervention groups, inclusion/exclusion criteria and outcomes. The study interventions were drawn from each record of the studies and linked to the ICF code and Core Sets for stroke and using the binding rules developed by Cieza et al. ${ }^{(12)}$. The comparisons between the collected data were made in the consensus meeting and the authors of articles were contacted, if necessary, to give some omitted information regarding the study.

\section{RESULTS}

Among the obtained results in the above-mentioned data bases, 125 articles were selected, in which 20 of them was found in PEDro, 5 in PubMed, 100 in the Web of Science, no records were found at Scielo. After applying the inclusion/exclusion criteria, there were 5 articles, as shown in Figure 1.

Table 1 presents, in summarized form, the methods and results of studies included in the present review, while in the table 2 shows the scales used in each study and the evaluated areas facing social participation, as well as a comparison of the Core Set of stroke areas related to social participation presented on each scale.

\section{DISCUSSION}

Social participation is considered a critic result of the successful rehabilitation ${ }^{(16)}$, however the inclusion of participation measures in randomized controlled trials in research on stroke are not often observed ${ }^{(17)}$ and the measuring tools of social participation are not consensual in the range areas which need to be addressed in post-stroke rehabilitation process $^{(18)}$. Even after the initial recovery period, which may include steps beyond time in rehab, recovering social roles and reintegrate back into the community may be very challenging for individuals who survives the stroke ${ }^{(19)}$.

The disabilities resulting from a stroke may lead to a variety of limitations and restrictions of participation. The ICF
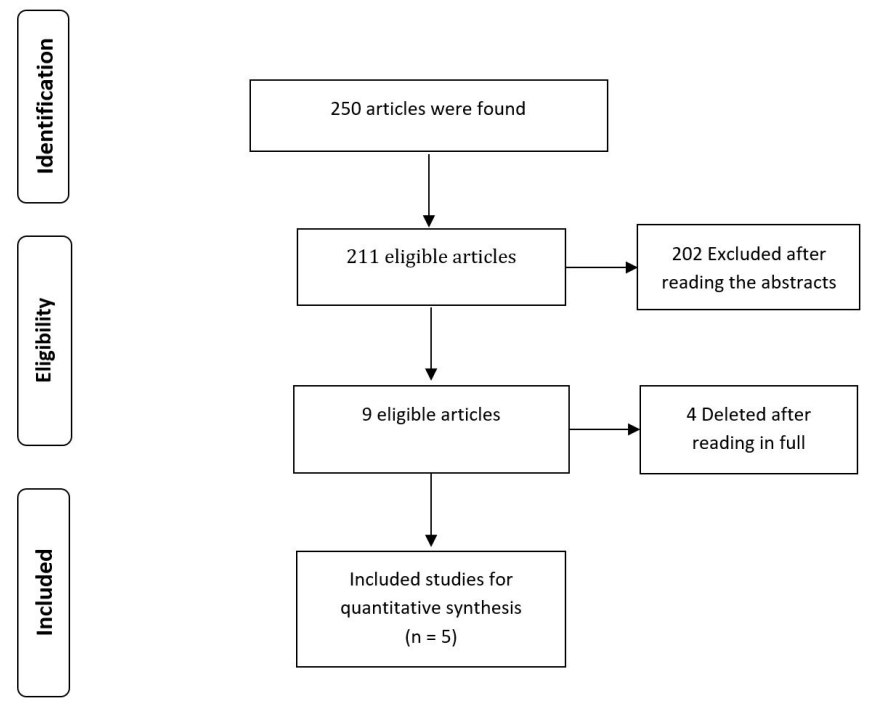

Figure 1. Selection process of included articles. 
Table 1. Research involving intervention and evaluation of social participation

\begin{tabular}{|c|c|c|}
\hline Authors, year. & Methods & Results \\
\hline Vincent-Onabajo, et al. $(2016)^{(6)}$ & $\begin{array}{l}\text { A multicenter study, conducted in Nigeria with } 96 \text { stroke } \\
\text { survivors. The social participation was assessed by the } \\
\text { Multidimensional Scale Perceived Social Support (MSPSS) } \\
\text { and London Handicap Scale (LHS). The LHS evaluated the } \\
\text { participation in six areas: mobility, physical independence, } \\
\text { social integration, occupation (career and leisure), } \\
\text { orientation and economic self-sufficiency. MSPSS assessed } \\
\text { the social support received from three sources, namely } \\
\text { "family," "friends," and "significant others." It consisted of } \\
12 \text { items, each item is scored on a 7-point scale ranging from } \\
1 \text { - "strongly disagree" to } 6 \text { - "agree very strongly". }\end{array}$ & $\begin{array}{l}\text { It was observed that in terms of participation, career } \\
\text { and leisure the participants presented less dominance, } \\
\text { which would not be directly related to a rehabilitation } \\
\text { program. }\end{array}$ \\
\hline Singam et al. $(2015)^{(10)}$ & $\begin{array}{l}\text { A total of } 121 \text { subjects were followed, initially assessed } \\
\text { while at the hospital unit soon after and } 5 \text { days after } \\
\text { the stroke. Subsequent, evaluations were performed } \\
\text { at } 3,6 \text { and } 12 \text { months, followed by } 6 \text { years post-stroke. } \\
\text { Participation was measured with Frenchay Activities } \\
\text { Index (FAI) containing } 15 \text { items representing three } \\
\text { areas: housekeeping, leisure/work and outdoor activities. }\end{array}$ & $\begin{array}{l}\text { It was observed that after } 6 \text { years, } 84 \% \text { of participants } \\
\text { could be described as active. The same level of } \\
\text { participation or better than the pre-stroke was found } \\
\text { in } 35 \% \text { of participants and in } 65 \% \text { the level was lower. }\end{array}$ \\
\hline
\end{tabular}
areas: housekeeping, leisure/work and outdoor activities.

It is a randomized, blind study with a sample of 26 subjects. All were submitted to a training program for four weeks after the pre-test. Post-test was performed three days after the end of the intervention period. The social participation was measured using a Stroke Impact Scale (SIS). The SIS is a self-administered questionnaire composed of a total of 64 questions in eight areas, including strength, the function of hands, the mobility and activities of daily living/instrumental activities of daily living, emotion, communication, memory and participation social.

Kim et al. $(2014)^{(13)}$

Lund et al. (2011)

Wang et al. (2015) $)^{(15)}$
This is a randomized controlled trial with a control group and an experimental group. The subjects aged over 65 years old, diagnosed with stroke were recruited from hospitals in Norway between June 2007 and December 2009. All subjects of the experimental group were submitted to 36 sessions of a lifestyle course and 36 of physical activity, and all from the control group performed 36 sessions of nonspecific physical activity.

Controlled, randomized, blind study (evaluator). Patients who had stroke were recruited from 3 university hospitals in southern Taiwan. The caregivers were paid to perform at The study shows that there is significant improvement in least two sessions of 60 to 90 minutes. The program was the rehabilitation and social participation process, and divided in three phases: Phase 1 (1-4 weeks), a program that intervention designed the conceptual model of ICF aimed at improving patient's body functions and structural and mediated by the family, improved the walking ability components; Phase 2 (5-8 weeks), a program designed to and integration in the community, as well as decreased improve the ability of patients to perform everyday activities the caregiver assistance to those patients. The SIS within their living environments using compensatory training showed an increase in scores after the rehabilitation methods and specific repairer; and Phase 3 (9-12 weeks), a program, statistically significant values. program designed to help patients to reintegrate to society by participating in recreational activities leisure. define participation as the participation in a life situation. Restrictions involving the social participation are classified as problems experienced by that involvement ${ }^{(20,21)}$. A aim of the rehabilitation is to return these people back to their earlier activities prior the pathology so they may have better quality of life ${ }^{(18,22)}$.

In a study by Vincent-Onabajo et al. ${ }^{(6)}$, it was observed that in terms of participation, career and leisure, the subjects had lower domain, which would not be directly related to a Rehabilitation program, since only $10 \%$ of the stroke survivors had involvement with occupational activities. When comparing the rating form study to the Core Set for these patients, it is clear that the participation is restricted and that the evaluated areas in the study are insufficient to determine the participation of these subjects.

Although the participation is accepted as a necessary outcome for rehabilitation, it requires a better understanding of the instruments designed for this type of outcome, particularly in terms of what is being evaluated, as it is being evaluated and if the evaluation tools are reliable and validated ${ }^{(9)}$.

Singam et al. ${ }^{(10)}$ presented in their study that the post-stroke individuals, six years after the event, returned to their level of 
Table 2. Correlation between the instruments in the evaluation of social participation and the items of social participation area of the Core Set for stroke.

Instruments of studies for the evaluation of social participation

orientation and economic self-sufficiency.
Correlation of Core Set domains for stroke - Social participation domain

D210 Performing a single task

D220 Performing various tasks

D230 Performing daily routine

D870 Economic self-sufficiency

D910 Community life

D920 Recreation and leisure

D175 Troubleshooting

D166 Reading

D210 Performing a single task

D230 Performing daily routine

D430 Lifting and transporting objects

D440 Fine use of hands

D445 Hand and arm use

Frenchay Activities Index (FAI) rating: 1-prepare main meals; 2- washing; 3- washing clothes; D450 Walking

4- light housework; 5- heavy housework; 6-local shopping; 7- social occasions; 8- walking outside D455 Moving

$>15$ min; 9- actively seeking hobby; 10 - driving car trip; 11- car ride; 12- gardening; 13- domestic car D460 Moving in different locations maintenance. 14. reading books; 15 - paid work.

D470 Using transportation

D475 Driving

D550 Eating

D640 Doing housework

D850 Paid work

D910 Community life

D920 Recreation and leisure

D175 Troubleshooting

D210 Performing a single task

D220 Performing various tasks

D230 Performing daily routine

D410 Changing the basic body position

Stroke Impact Scale (SIS) contains 59 items which measure 8 areas: strength, hand function, activities of D415 Maintain a position of the body

daily living/instrumental activities of daily living, mobility, communication, emotion, memory/thinking D420 Moving

and social participation.
D440 Fine use of hands

D450 Walking

D455 Moving

D550 Eating

D640 Doing housework

D860 Basic Economic Transactions

D210 Performing a single task

D220 Performing several tasks

D230 Performing daily routine

D240 Stress management and other psychological

demands

D420 Moving

D430 Lifting and transporting objects

D440 Fine use of hands

D450 Walking

D510 Wash off

D350 Conversation

D710 Basic interpersonal interactions

D760 family relationships

The Multidimensional Scale Perceived Social Support consists of 12 items and assesses the received social support from three sources, namely "family," "friends" and "significant others".

besides social participation. These instruments were incapable of encoding the full experience of post-stroke people by not considering, in full, non-physical abilities. Corroborating this statement, Hardie et al. ${ }^{(23)}$ states that, despite $64 \%$ of stroke patients have limited social participation in employment, in the daily living and leisure activities, the most rehabilitation approaches in this population mainly considers the functional assessments or those related to activities. According to Crawford et al. ${ }^{(24)}$ the evaluation of social participation is 
important in stroke rehabilitation and it should be included as a routine part of the rehabilitation. Corroborating with this statement, Kim et al. ${ }^{(13)}$ had reported on the assessment and care with the social participation, however the results were not displayed properly.

The only study which used in its program, a designed model based on ICF and an intervention mediated by the family, it was the study of Wang et al. ${ }^{(15)}$. They observed that there was a good functional recovery and social reintegration of individuals and key contributing factor to this was the adherence to the conceptual framework of the ICF, in which emphasizes family involvement, training and repetitive tasks and the reintegration into society. This result demonstrated clearly that a rehabilitation program designed based on the conceptual framework emphasizing the repetitive training at home, outdoor activities and social reintegration is effective in improving the functional recovery of post-stroke patients.

\section{CONCLUSION}

It is suggested that future studies direct their interventions to improve specific aspects of social participation, including the involvement of the individual in activities which provide interaction with the community and the society. In addition, outcome measures that measure the multiple characteristics of social participation, such as social contacts and contributions to society, should be used. Studies are needed to determine the optimal rehabilitation strategy aimed at improving social participation.

\section{AUTHOR'S CONTRIBUTION}

FHNS, EMSF, LGC, ELAM, EWAC contributed equally to the design of the study, the analysis and data interpretation and the preparation of the manuscript. All also approved the final version to be published.

\section{CONFLICTS OF INTEREST}

The author(s) declare that they have no competing interests.

\section{REFERENCES}

1. Haas B, Playford ED, Ahmad AQ, Yildiran T, Gibbon AJ, Freeman JA. Rehabilitation goals of people with spinal cord injuries can be classified against the International Classification of Functioning, Disability and Health Core Set for spinal cord injuries. Spinal Cord. 2015;54(4):324-28.

2. Obembe AO, Eng JJ. Rehabilitation interventions for improving social participation after stroke: a systematic review and metaanalysis. Neurorehabilitation and neural repair. 2015;30(4):384-92.

3. Carpenter C, Forwell SJ, Jongbloed LE, Backman CL. Community participation after spinal cord injury. Archives of physical medicine and rehabilitation. 2007;88(4):427-33.

4. Hartman-Maeir A, Soroker N, Ring H, Avni N, Katz N. Activities, participation and satisfaction one-year post stroke. Disability and rehabilitation. 2007;29(7):559-66.

5. Schepers VP, Ketelaar M, Van de port IG, Visser-Meily E, Lindeman $J M$. Comparing contents of functional outcome measures in stroke rehabilitation using the international classification of functioning, disability and health. Disability and Rehabilitation. 2007;29(3):221-30.
6. Vincent-Onabajo GO, Ihaza LO, Ali MU, Masta MA, Majidadi R, Modu A et al. Impact of social support on participation after stroke in Nigeria. Topics in stroke rehabilitation. 2016;23(5):305-10.

7. Geyh S, Cieza A, Schouten J, Dickson H, Frommelt P, Omar Z et al. Core sets for stroke. Journal of rehabilitation medicine. 2004;44:135-41.

8. Rauch A, Cieza A, Stucki G. How to apply the international classification of functioning, disabilityand health (ICF) for rehabilitation management in clinical practice. Eur J Phys Rehabil Med. 2008;44(3):329-42.

9. Tse T, Douglas J, Lentin P, Carey L. Measuring participation after stroke: a review of frequently used tools. Archives of physical medicine and rehabilitation. 2013;94(1):177-92.

10. Singam A, Ytterberg C, Tham K, Koch L. Participation in complex and social everyday activities six years after stroke: predictors for return to pre-stroke level. Plos One. 2015;10(12).

11. Shamseer L, Moher D, Clarke M, Ghersi D, Liberati A, Petticrew M et al. PRISMA-P Group. Preferred reporting items for systematic review and meta-analysis protocols (PRISMA-P) 2015: elaboration and explanation. BMJ. 2015;2:349.

12. Cieza A, Geyh S, Chatterji S, Kostanjsek N, Ustün B, Stucki G. ICF linking rules: an update based on lessons learned. J Rehabil Med. 2005;37(4):21218.

13. Kim M, Cho K, Lee W. Community walking training program improves walking function and social participation in chronic stroke patients. The tohoku journal of experimental medicine. 2014;234(4):281-6.

14. Lund A, Michelet M, Sandvik L, Wyller TB, Sveen U. A lifestyle intervention as supplement to a physical activity programme in rehabilitation after stroke: a randomized controlled trial. Clinical Rehabilitation. 2011;26(6):502-12.

15. Wang TC, Tsai AC, Wang JY, Lin WT, Lin KL, Chen JJ et al. Caregiver-mediated intervention can improve physical functional recovery of patients with chronic stroke: a randomized controlled trial. Neurorehabilitation and neural repair. 2014;29(1):3-12.

16. Desrosiers J. Muriel Driver Memorial Lecture. Participation and occupation. Canadian Journal occupational therapy. 2005;1(72):195-204.

17. Salter K, Foley N, Jutai J, Teasell R. Assessment of participation outcomes in randomized controlled trials of stroke rehabilitation interventions. International journal of rehabilitation research. 2007;30(4):339-42.

18. Salter K, Jutai J, Teasell R, Foley N, Bitensky J, Bayley M. Issues for selection of outcome measures in stroke rehabilitation: ICF participation. Disability and rehabilitation. 2005;27(9):507-28.

19. Tavener M, Thijsen A, Hubbard IJ, Francis JL, Grennall C, Levi C Acknowledging how older Australian women experience life after stroke: how does the WHO 18-item brief ICF core set for stroke compare? Health care for women international. 2015;36(12):1311-26.

20. World Health Organization - WHO. International classification of functioning, disability and health. Geneva: World Health Organization; 2001.

21. Zhang L, Yan T, You L, Li K. Barriers to activity and participation for stroke survivors in rural China. Archives of physical medicine and rehabilitation. 2015;96(7):1222-8.

22. Whiteneck GG. Issues affecting the selection of participation measurement in outcomes research and clinical trials. Archives of physical medicine and rehabilitation. 2010;91(9):54-9.

23. Hardie K, Hankey GJ, Jamrozik K, Broadhurst RJ, Anderson C. Ten-year risk of first recurrent stroke and disability after first-ever stroke in the Perth community stroke study. Stroke. 2004;35(1):731-5.

24. Crawford A, Hollingsworth HH, Morgan K, Gray DB. People with mobility impairments: physical activity and quality of participation. Disabil Health J. $2008 ; 1(1): 7-13$. 